\title{
Criminologie
}

\section{Les décisions pénales dans les causes d'homicide au tribunal de Montréal, de 1985 à 1989}

\section{Rachel Noël-Grandmaison}

Volume 29, numéro 1, printemps 1996

Intervenir auprès des délinquants

URI : https://id.erudit.org/iderudit/017385ar

DOI : https://doi.org/10.7202/017385ar

Aller au sommaire du numéro

\section{Éditeur(s)}

Les Presses de l'Université de Montréal

ISSN

0316-0041 (imprimé)

1492-1367 (numérique)

Découvrir la revue

Citer cet article

Noël-Grandmaison, R. (1996). Les décisions pénales dans les causes d'homicide au tribunal de Montréal, de 1985 à 1989. Criminologie, 29(1), 141-160.

https://doi.org/10.7202/017385ar
Résumé de l'article

This paper tries to shed a light on the attrition process and on the factors influencing the sentencing's decision making process, for criminal homicide cases prosecuted in Montreal between 1985 and 1989 inclusively. The results showed that unlike the police mainly laying murder charges, $55 \%$ of the 142 sentenced were found guilty of manslaughter. Therefore a large number has less than eight years in prison to serve before eligibility to full parole. The accused who commited an homicide associated with another crime or a settling of accounts, who went through a trial, who had a criminal record and who killed a woman significantly encounter a greater possibility to be sentenced on a murder charge. Yet a sequence in the decision making process can be observed: once is settled the type of charge to be definitive at the end of the criminal process, the factors studied influence rather little the severity of the imposed sentence. Finally the author points out how the prosecution mode of these homicide cases played an important part on the two levels of decision. 
LES DÉCISIONS PÉNALES DANS LES CAUSES D'HOMICIDE

AU TRIBUNAL DE MONTRÉAL, DE 1985 À 1989

Rachel Noël-Grandmaison ${ }^{1}$

This paper tries to shed a light on the attrition process and on the factors influencing the sentencing's decision making process, for criminal homicide cases prosecuted in Montreal between 1985 and 1989 inclusively. The results showed that unlike the police mainly laying murder charges, $55 \%$ of the 142 sentenced were found guilty of manslaughter. Therefore a large number has less than eight years in prison to serve before eligibility to full parole. The accused who commited an homicide associated with another crime or a settling of accounts, who went through a trial, who had a criminal record and who killed a woman significantly encounter a greater possibility to be sentenced on a murder charge. Yet a sequence in the decision making process can be observed: once is settled the type of charge to be definitive at the end of the criminal process, the factors studied influence rather little the severity of the imposed sentence. Finally the author points out how the prosecution mode of these homicide cases played an important part on the two levels of decision.

\section{INTRODUCTION}

La recherche portant sur le fonctionnement de la justice s'est peu penché sur les décisions prises par les intervenants judiciaires dans les causes d'homicide de responsabilité criminelle. Au Canada, il est surprenant de constater que si peu de données sur cette question soient disponibles. Ce que nous savons par ailleurs, c'est qu'il existe un écart entre les premières accusations portées par la police, le plus souvent des accusations de meurtre, et celles portées à la conclusion des procédures (Boyd, 1988; Daly et Wilson, 1988), souvent d'accusations d'homicides involontaires.

La présente étude vise à combler un tel vide en cherchant à mieux comprendre l'intervention du tribunal de Montréal dans les causes d'homicide $^{2}$. Deux objectifs spécifiques ont été poursuivis : cerner le processus d'attrition des inculpés au cours des procédures judiciaires suivies au

1. Centre international de criminologie comparée, École de criminologie, Université de Montréal.

2. Cet article reprend les éléments essentiels d'un mémoire de mâtrise en criminologie du même titre déposé en décembre 1993. L'auteure tient à exprimer de sincères remerciements à M. Maurice Cusson, Ph.D., ainsi qu'à M. Marc Ouimet, Ph.D., pour leurs précieux conseils. 
tribunal et identifier les facteurs influençant les décisions pénales prises à leur endroit, en l'occurrence ce qui influence la sévérité de la peine imposée.

Appliqué en justice criminelle, le processus d'attrition indique la proportion d'accusés qui sont soit rejetés, soit inclus dans certaines catégories au cours des différentes étapes du processus judiciaire. Le fait d'être en mesure de cerner le cheminement judiciaire des accusés nous permet de mieux saisir à quelles étapes sont prises les décisions cruciales pour l'issue pénale des causes. Vue de cette façon, l'imposition d'une sentence devient la conséquence des décisions et des interactions de l'ensemble des acteurs judiciaires au cours de ces étapes (Blumstein et coll., 1983; Cousineau, 1992).

Dans un autre ordre d'idées, la recherche des facteurs affectant la sévérité des sentences criminelles demeure une préoccupation qui continue de soulever des controverses (voir entre autres Stipak et McDavid, 1983; McCarthy et Lindquist, 1984). Selon McCarthy et Lindquist (1984, p. 37), les analyses ne différenciant pas les types de crimes masquent d'importantes corrélations ou en génèrent d'autres qui ne correspondent pas à la réalité de certains crimes spécifiques, en l'occurrence en ce qui a trait aux crimes violents. En ce sens, il est donc approprié de ne s'attarder qu'à l'étude distincte des facteurs influençant la sévérité des peines rendues dans le cas de l'homicide.

\section{L'HOMICIDE : LES TYPES DE CRIMES ÉTUDIÉS}

Les différentes infractions susceptibles de se rencontrer lors des premières accusations portées envers un individu soupçonné d'être l'auteur d'un homicide sont le meurtre au premier et au second degré, l'homicide involontaire coupable et l'infanticide ${ }^{3}$ tels que définis et proscrits aux articles 222 à 240 du Code criminel canadien (Dubois, 1989).

Pour résumer, le fait d'accuser une personne de meurtre, à la différence de l'homicide involontaire coupable, nécessite qu'on ait fait la preuve de I'intention de tuer la victime (Dubois, 1989; Gravel et Baril, 1990) ${ }^{4}$. Le meurtre au premier degré se distingue par le fait que: a) l'acte a été prémédité ou délibéré; b) la victime est une personne qui a été engagée et

3. Il ne sera pas question de l'infanticide puisque aucune cause au tribunal de Montréal entre 1985 et 1989 n'a concerné ce chef d'accusation.

4. Cependant, avant 1990, la notion d'intention par interprétation (constructive intent) permettait de qualifier de meurtre le fait de causer la mort d'une personne au cours de la commission ou de la tentative de commission d'une infraction criminelle grave telle le vol qualifié, que l'intention de tuer cette personne ait été présente ou non (Commission de réforme du droit du Canada, 1984; Dubois, 1989; Silverman et Kennedy, 1993). 
qui exerçait ses fonctions ; c) l'acte est survenu durant la perpétration d'une infraction criminelle grave particulière, que l'intention de causer la mort d'autrui ait été présente ou non, et finalement d) l'auteur a déjà été déclaré coupable de meurtre (abrogé en 1989; voir la Commission de réforme du droit du Canada, 1984; Dubois, 1989). Tout meurtre qui n'appartient pas à l'une ou l'autre des catégories du meurtre au premier degré est un meurtre au deuxième degré; ce chef d'accusation est plutôt résiduel et n'est pas clairement défini par le Code criminel (Silverman et Kennedy, 1993). Enfin, l'homicide involontaire coupable est considéré, en général, comme un homicide commis dans un accès de colère, à la suite d'une provocation soudaine. Pour être admissible au tribunal à titre de défense, la provocation doit être telle que toute personne raisonnable perdrait le contrôle d'ellemême en une situation semblable. Cependant, elle ne doit pas être invoquée si l'agresseur a poussé la victime à la provocation (Commission de réforme du droit du Canada, 1984 ; Dubois, 1989 ; Côté, 1991).

La peine encourue pour meurtre, qui consiste en une peine minimale obligatoire, est l'emprisonnement à perpétuité. Dans le cas du meurtre au premier degré, la période d'incarcération à purger avant l'admissibilité à une libération conditionnelle totale s'élève à 25 ans. Pour le meurtre au deuxième degré, ce délai correspond à l'accomplissement d'au moins dix ans de la peine jusqu'à concurrence de 25 ans en vertu de l'article 744 du Code criminel. Si toutefois l'individu a déjà été condamné dans le passé pour homicide coupable équivalent à un meurtre, il devra purger 25 ans (art. 742 C.c.). Par ailleurs, tout condamné ayant purgé quinze ans de sa peine peut demander à ce que soit réduit le délai préalable à sa libération conditionnelle par un processus de révision par jury (art. 745 C.c.). En ce qui concerne l'homicide involontaire coupable, la peine maximale prévue par le Code criminel est l'emprisonnement à perpétuité; aucune peine minimale obligatoire ne régit cette infraction (Commission de réforme du droit du Canada, 1984; Dubois, 1989). La loi sur les libérations conditionnelles prévoit qu'un individu condamné sur cette accusation à une peine d'emprisonnement de six mois ou plus peut être admissible à une libération conditionnelle totale dès le tiers de la peine purgée, jusqu'à concurrence de sept ans (Québec, 1989; Canada, 1990).

\section{MÉTHODOLOGIE}

Cette étude a êté réalisée à l'aide de la banque de données développée dans le cadre d'une recherche plus vaste sur l'homicide au Québec, sous la direction de M. Maurice Cusson de l'École de criminologie de l'Université de Montréal. Compte tenu des objectifs poursuivis, nous avons choisi la méthodologie quantitative. 
La cueillette de données a permis de répertorier et d'analyser l'ensemble des homicides, tels que définis ci-haut, qui se sont produits sur l'île de Montréal de 1985 à 1989 inclusivement. Un formulaire d'enquête abordant plusieurs volets tels les caractéristiques de l'événement, celles de la victime et de l'agresseur ainsi que les procédures judiciaires concernant l'agresseur fut rempli pour chaque homicide. Lorsque plus d'un meurtrier était dénombré dans un même événement, un questionnaire séparé était rempli pour chacun des complices.

Deux sources de données furent compilées, soit le journal Allô Police, qui est un hebdomadaire montréalais spécialisé dans les chroniques sur les homicides, et les dossiers du tribunal. À partir du bilan annuel d'Allô Police, tous les homicides commis sur l'île de Montréal qui y étaient relatés furent relevés et firent l'objet d'une recherche à même les chroniques. Des informations étaient recherchés tout au plus un an après l'ouverture de l'enquête policière pour chacune des affaires. Comme seconde source de données, les dossiers du Palais de justice de Montréal foumirent des informations pertinentes en ce qui concerne les procédures judiciaires suivies et la sentence prononcée. Divers documents se retrouvent dans ces dossiers, dont voici les principaux : l'acte d'accusation, le procès-verbal de chacune des procédures, et la déclaration de culpabilité. Parfois, on y trouve aussi d'autres documents tels le rapport des antécédents judiciaires de l'accusé provenant du Service de l'identité judiciaire de la Gendarmerie royale du Canada ou le rapport sur la décision sentencielle du juge.

La lecture des documents contenus dans les dossiers de la Cour ont permis de vérifier si les données recueillies correspondaient au contenu des articles parus dans le journal Allô Police. Ceci dit, les informations communiquées par ce journal concernant les affaires d'homicide sont relativement fiables. Cordeau (1989, p. 15) s'était déjà prononcé en ce sens, spécifiant qu'au-delà du sensationnalisme des mots, les informations factuelles recueillies par ce journal se révèlent fiables et conformes à celles des archives policières.

Finalement, spécifions que la banque de données initialement conçue sur la base des victimisations fut transformée sur la base des agresseurs. Cette opération s'est avérée nécessaire pour pouvoir suivre chaque cause à travers le processus judiciaire. Au total, 185 individus âgés de 18 ans ou plus soupçonnés d'être les auteurs d'un homicide ont été poursuivis en justice pendant la période à l'étude.

\section{Le cheminement des accusés à travers le processus judiciaire}

Nous reprenons ici les principales étapes du cheminement judiciaire que suivirent ces 185 individus impliqués dans des homicides entre 1985 et 
1989, et qui furent traduits devant le tribunal de Montréal, ce que nous avions décrit plus tôt comme le processus d'attrition des cas 5 .

\section{I La comparution}

Lors de la comparution, le procureur de la Couronne doit émettre un acte d'accusation et déterminer quels doivent être les chefs d'accusation reprochés à un individu soupçonné d'être l'auteur d'une infraction criminelle. Il ressort que dans les causes d'homicide, la Couronne choisit avant tout de porter des accusations de meurtre au premier degré ainsi qu'au second degré (respectivement, $46 \%$ et $41 \%$ des cas). Les autres sujets sont accusés d'homicide involontaire coupable $(13,4 \%)$.

Gravel et Baril (1990, p. 165) constatent également que les accusations originales de meurtre sont plus fréquentes que celles d'homicide involontaire coupable. Au Canada, dans l'ensemble, la police aurait tendance dès le départ à décrire plus ou moins $90 \%$ des homicides comme des meurtres (Boyd, 1988; Statistique Canada, 1990).

\subsection{L'enquête préliminaire}

En matière de crimes graves, il se tient habituellement une enquête préliminaire en vertu de laquelle la Couronne divulgue une partie de sa preuve, permettant ainsi au juge d'évaluer, d'une part, s'il y a matière à procès et, d'autre part, si les accusations imputées au contrevenant sont justes (Nakell et Hardy, 1987; Gravel et Baril, 1990). Suite à cette étape, nous remarquons que des modifications au plan des accusations se produisent en particulier chez les causes initialement de meurtre au premier et au second degré. Ainsi, à ce stade des procédures, le nombre d'individus inculpés d'homicide involontaire coupable s'est majoré, passant de 24 à 40 . Il demeure toutefois que $75 \%$ des sujets sont toujours accusés de meurtre.

Cette situation est compréhensible en raison non seulement des éléments de preuve requis, mais de la volonté de la défense, dans certains cas, de négocier une diminution de la gravité qualitative de l'accusation. Cependant, il se pourrait que, dans certaines affaires, le procureur de la Couronne ait émis à l'origine des accusations plus graves que ne le justifierait le crime (Gravel et Baril, 1990).

Spécifions que huit accusés avaient dès lors complété leur cheminement. En effet, trois accusés se virent libérer au moment de leur enquête préliminaire, quatre autres enregistrèrent un plaidoyer de culpabilité avant ou pendant l'enquête préliminaire et, enfin, un accusé décéda en cours de procédures.

5. Le processus d'attrition des cas est représenté par un schéma dans le mémoire de maîtrise. 


\subsection{L'issue judiciaire}

Les possibilités quant à l'issue judiciaire finale des causes d'homicide étudiées vont de la libération de l'accusé en passant par un plaidoyer de culpabilité jusqu'à un verdict de culpabilité ou à un acquittement suite à un procès.

À l'issue des procédures, ce sont non moins de $80 \%$ des accusés, soit 142 sur 177, qui se virent déclarer coupables des crimes qui leur étaient reprochés. Parmi les sujets déclarés coupables, $37 \%$ ont enregistré un plaidoyer de culpabilité à l'accusation portée par la Couronne, tandis que $32 \%$ plaidèrent coupables à une accusation moindre ou différente. Les derniers $31 \%$ ont été reconnus coupables suite à la tenue d'un procès.

La proportion de causes d'homicide acheminées au tribunal qui se terminèrent par un plaidoyer de culpabilité est de près de $56 \%$. Bien que nous puissions en déduire qu'il existe une pratique courante de négociation entre avocats relative aux dossiers d'homicide, cette proportion semble plus faible qu'on ne l'a dit. Elle se situe entre 70 et $90 \%$ selon ce que constate Poirier (1987, p. 57), tandis que Cousineau (1992, p. 431) arrive à un taux de $65,5 \%$. Figueira-McDonough (1985) avance l'hypothèse que les individus accusés de crimes graves sont plus enclins à clamer leur innocence. En ce sens, McCarthy et Lindquist (1984, p. 41) concluent que la plupart des individus accusés de meurtre maintiennent leur plaidoyer initial de nonculpabilité tout au long du processus judiciaire.

En ce qui concerne les individus inculpés qui choisirent d'aller en procès, près des deux tiers ( 44 cas sur 71 ) durent composer avec un verdict de culpabilité. Par conséquent, très peu d'accusés ont été acquittés suite à un procès. La majorité des acquittements, quelle que soit leur nature, proviennent des causes initialement de meurtre au premier et au second degré. Or, il semble qu'il y aurait un lien directement proportionnel entre la sévérité de la peine prescrite pour un crime et la quantité de preuve requise afin qu'un verdict de culpabilité soit rendu. De surcroît, plus la peine prescrite est sévère, plus les probabilités de condamnation auraient tendance à diminuer (Kerr, 1978). Toutefois, les données de notre étude ne nous permettent pas de nous prononcer sur la validité de la preuve.

L'accusation décisive lors du jugement de culpabilité est principalement l'homicide involontaire coupable $(55 \%)$, suivie de l'accusation de meurtre au second degré, puis de celle de meurtre au premier degré (respectivement, $33,1 \%$ et $7,8 \%$ ). Pour terminer, quelques accusés ont été déclarés coupables sur des accusations différentes de celles qui prévalent en matière de crimes d'homicide. En ce sens, nous avons observé qu'une partie importante des sujets plaidèrent coupable à une accusation moindre que celle initialement portée. De plus, ceux ayant été reconnus coupables suite à un 
procès peuvent aussi avoir bénéficié d'une réduction de la gravité de l'accusation; à l'occasion, le jury condamne le prévenu sur un chef de moindre gravité après avoir entendu les instructions du juge et évalué les preuves.

Dans sa monographie portant sur le meurtre au Canada, Boyd (1988, p. 19) constate des proportions similaires aux nôtres, avec un peu plus de $60 \%$ des accusés condamnés pour homicide involontaire coupable. Ainsi, le nombre d'accusés déclarés coupables de meurtre s'avère inversement proportionnel à celui du nombre de prévenus qui étaient, à l'origine, accusés de cette infraction (Boyd, 1988 ; Côté, 1991).

\subsection{Les sentences imposées}

Les résultats en ce qui a trait à la peine prononcée correspondent à la dernière sentence imposée, soit celle qui se veut décisive peu importe l'instance qui la rend.

Au total, 133 des 142 individus déclarés coupables ont été condamnés à une sentence carcérale; celle-ci s'accompagna parfois d'une autre peine telle l'interdiction de port d'armes durant cing ou dix ans. La moitié d'entre eux ( $53 \%)$ ont été condamnés à une peine autre qu'à perpétuité. Bien qu'ils aient en moyenne près de huit années d'emprisonnement à purger, on retrouve des peines allant d'aussi peu que six mois à 20 ans d'emprisonnement. Les autres sujets, 63 au total, ont été condamnés à l'emprisonnement à perpétuité. Près de la moitié d'entre eux ont la possibilité d'être libérés dès qu'ils auront purgé pour certains au moins sept années d'emprisonnement (homicide involontaire coupable), pour la plupart au moins dix années (meurtre au second degré). Cependant, 12 individus, ou 8,5\% des sujets ayant eu une sentence, reçurent la peine la plus lourde prescrite par le Code criminel, soit 25 ans avant toute possibilité de libération conditionnelle totale.

Afin de réaliser des analyses explicatives, l'ensemble des sentences, de types et de quanta différents, ont été disposées sur une seule et même échelle de gradation de la sévérité. Celle-ci comprend une unité de mesure en nombre d'années d'incarcération, ce qui en fait une échelle proportionnelle continue. Pour effectuer la standardisation, la base de calcul adoptée dans le cas des peines carcérales fut le délai minimal prévu avant l'éligibilité à une libération conditionnelle totale. Soulignons que n'est pas considérée la procédure voulant qu'un détenu puisse faire une demande de révision du délai après quinze ans d'incarcération. Nous n'avons pas de données sur l'application de cette procédure.

Les équivalences pénales relevées de la métrique pénale (voir Tremblay, Gravel et Cusson, 1987) furent utilisées dans le cas des peines non carcérales, qui sont peu nombreuses dans cette étude. Pour la majorité, 
elles impliquent une libération conditionnelle ou une sentence suspendue accompagnée de trois années de probation. Cette période de probation équivaut à 1,3 mois d'emprisonnement ferme, donc à 0,11 année d'emprisonnement, selon les conclusions avancées par Tremblay, Gravel et Cusson $(1987, \text { p. } 86)^{6}$.

\section{Tableau 1}

Statistiques descriptives concernant le délai minimal prévu avant l'éligibilité à une libération conditionnelle totale, en nombre d'années, pour l'ensemble des individus condamnés

\begin{tabular}{|llllll|}
\hline Moyenne & 7,64 & Médiane & 4,17 & Mode & 10,00 \\
Écart type & 7,33 & Minimum & $\mathbf{0 , 0 1 *}$ & Maximum & $\mathbf{2 5 , 0 0}$ \\
Valeurs présentes & $\mathbf{1 4 2}$ & & & & \\
\hline
\end{tabular}

* Individu condamné à une sentence suspendue sans ordonnance de probation. Ce score le différencie d'un individu qui aurait été acquitté.

Comme l'indique le tableau 1, la moitié des sujets condamnés ont quatre ans d'emprisonnement ou moins à purger avant d'être admissibles à une libération conditionnelle totale; $60 \%$ ont même moins de huit ans à purger. Cette situation est due au nombre important de sujets déclarés coupables d'homicide involontaire coupable pour lesquels, nous le savons, il est possible d'être libérés après, au maximum, sept ans d'emprisonnement. Finalement, nous observons 26 sujets condamnés à l'emprisonnement à perpétuité dont le délai avant de pouvoir effectuer une demande de libération conditionnelle totale a été fixé à dix ans.

\section{Les facteurs qui influencent la sévérité de la peine}

Comme nous l'avons constaté, nombreux sont les dossiers d'homicide traités au tribunal de Montréal entre 1985 et 1989 pour lesquels l'auteur ou au moins un auteur a été déclaré coupable et s'est vu imposer une peine relativement à un homicide auquel il avait pris part. Cette deuxième section a pour buit d'étudier l'influence d'un certain nombre de facteurs sur la sévérité de la peine prononcée. Cependant, en matière d'homicide, la sévérité de la sentence résulte en grande partie du chef d'accusation sur lequel porte le jugement de culpabilité, puisque le Code criminel stipule qu'une peine minimale obligatoire d'emprisonnement à perpétuité doit être imposée

6. Blumstein et ses collègues (1983, p. 11) ont souligné les limites engendrées par l'utilisation d'une échelle arbitraire qui combine ainsi différents types de sentences, rendant difficile l'évaluation de l'impact de divers facteurs sur chacun des types. Toutefois, notre échelle reflète une homogénéité importante en ce qui a trait à la nature de la sentence, très peu de contrevenants condamnés pour homicide ayant eu à purger une sentence non carcérale. 
aux individus condamnés pour meurtre. En conséquence, les analyses effectuées seront contrôlées par cette variable qu'est l'accusation sur laquelle porte le verdict de culpabilité. Du même coup, nous examinerons s'il existe une association entre cette dernière variable et chacun des facteurs.

Tout en n'écartant pas le fait qu'il puisse se produire une évaluation de la gravité à tout le moins subjective des homicides, les analyses effectuées se rapportent à une multitude de facteurs plus concrets. En justice criminelle, l'on soutient communément que la sentence imposée devrait refléter les faits de la cause, le dossier criminel de l'accusé les circonstances aggravantes ou atténuantes (voir entre autres Nadin-Davis, 1982; Ruby, 1987). D'autres facteurs pourraient être considérés comme ayant un effet davantage illégitime sur la sentence, tels les caractéristiques du contrevenant et celles de la victime ou d'autres éléments émanant du déroulement des procédures à la cour.

Le tableau 2 présente les résultats aux analyses bivariées. Les facteurs, regroupés conceptuellement, sont définis ci-bas. Ce tableau, qui comprend un total de 135 agresseurs condamnés pour meurtre ou homicide involontaire coupable, indique la mesure de l'influence de chacun des facteurs sur la sévérité de la peine prononcée par l'entremise de la durée d'emprisonnement à purger préalablement à l'admissibilité à une libération conditionnelle totale. Bien que le délai d'emprisonnement moyen global s'établisse à huit années d'emprisonnement, les délais moyens diffèrent beaucoup selon qu'il s'agisse d'une accusation finale de meurtre au premier degré (automatiquement 25 ans), de meurtre au second degré (12,6 ans), ou d'homicide involontaire coupable (2,7 ans). Cette différence remarquable entre les délais moyens globaux du meurtre et de l'homicide involontaire coupable fut déjà soulevée par des auteurs tels Nadin-Davis (1982) et Ruby (1987).

Dans le premier groupe de facteurs, nous avons retenu les signes de préméditation, qui rejoignent la question de l'intention criminelle de tuer autrui dans les cas de meurtre. Cette variable fut codifiée d'après les renseignements fournis par Allô Police. Par conséquent, ceux-ci ne permettent pas de se prononcer sur la validité de la preuve à ce propos. Comme second facteur, les déclencheurs immédiats sont des indices d'une quelconque provocation de la part de la victime. Un déclencheur immédiat se définit comme une action posée par la victime durant les six heures précédant l'homicide, et dont on pense qu'elle ne peut manquer d'engendrer de la frustration chez l'agresseur. L'opérationnalisation de cette variable a donc un sens plus large qu'aux termes de la loi. Les antécédents judiciaires, à savoir si l'agresseur avait déjà été reconnu coupable dans le passé pour la commission d'au moins une infraction criminelle quelconque, est une variable faisant également partie de ce regroupement. Malgré les biais que ce choix entraîne, nous ne considérerons que la présence ou l'absence 
Tableau 2

L'influence d'une série de facteurs sur la sévérité de la peine prononcée en fonction de l'accusation sur laquelle porte le jugement

\begin{tabular}{|c|c|c|c|c|}
\hline Var. indépendantes & M 1 $\mathbf{e r}^{\mathrm{e}}$ degré & M $2^{\mathrm{e}}$ degré & H.I.C. & Total \\
\hline \multicolumn{5}{|c|}{ Signes de préméditation** } \\
\hline Oui & $25,00(n=6)$ & $11,53(n-17)$ & $3,21(n-16)$ & $10,19(n=39)$ \\
\hline \multirow[t]{2}{*}{ Non } & $25,00(n=3)$ & $13,67(n=21)$ & $2,53(n-50)$ & $6,60(n-74)^{* *}$ \\
\hline & & & & $16,3 \%^{\mathrm{a}}$ \\
\hline \multicolumn{5}{|l|}{ Déclencheur immédiat** } \\
\hline Oui & $25,00(n=2)$ & $12,39(n-23)$ & $2,70(n=49)$ & $6,31(n=74)$ \\
\hline \multirow[t]{2}{*}{ Non } & $25,00(\mathrm{n}=8)$ & $13,77(n-17)$ & $2,55(n=20)$ & $10,78(n=45)^{* * *}$ \\
\hline & & & & $11,9 \%$ \\
\hline \multicolumn{5}{|c|}{ Antécédents judiciaires** } \\
\hline Ouj & $25,00(n=8)$ & $13,41(n-34)$ & $2,95(n-39)$ & $9,52(n=81)$ \\
\hline \multirow[t]{2}{*}{ Non } & $25,00(n=1)$ & $10,00(n-6)^{b}$ & $2,27(n=28)$ & $4,24(n=35)^{* * *}$ \\
\hline & & & & $14,1 \%$ \\
\hline \multicolumn{5}{|l|}{ Arme à feu*** } \\
\hline Oui & $25.00(n=7)$ & $12,14(n=7)$ & $2,95(n-24)$ & $8,71(n-38)$ \\
\hline \multirow[t]{2}{*}{ Non } & $25,00(n=4)$ & $12,73(n=40)$ & $2,65(n=53)$ & $7,72(n=97)$ \\
\hline & & & & - \\
\hline \multicolumn{5}{|l|}{ Genre de l'agresseur } \\
\hline Masculin & $25,00(n=11)$ & $12.70(n=46)$ & $2,80(n-70)$ & $8,31(n=127)$ \\
\hline \multirow[t]{2}{*}{ Féminin } & - & $10,00(n=1)$ & $1,96(n=6)^{C}$ & $3,11(n-7)$ \\
\hline & & & & $0,7 \%$ \\
\hline \multicolumn{5}{|l|}{ Genre de la victime } \\
\hline Masculin & $25,00(n-7)$ & $12,83(n=23)$ & $2,71(n-47)$ & $7,76(n=77)$ \\
\hline \multirow[t]{2}{*}{ Féminin } & $25,00(n=2)$ & $12,05(n=21)$ & $2,78(n=27)$ & $7,56(n=50)$ \\
\hline & & & & $5,9 \%$ \\
\hline \multicolumn{5}{|c|}{ Rel. entre protagoninistes* } \\
\hline Aucune & $25,00(n=3)$ & $13,71(n=14)$ & $2,54(n=10)$ & $10,83(n=27)$ \\
\hline-24 heures & - & $12,60(n=5)$ & $2,19(n=4)$ & $7,98(n=9)$ \\
\hline \multirow[t]{2}{*}{+24 heures } & $25,00(n=6)$ & $11,59(n=22)$ & $2,75(n-57)$ & $6,61(n=85)^{*}$ \\
\hline & & & & $10,4 \%$ \\
\hline \multicolumn{5}{|l|}{ Type d'homicide*** } \\
\hline Fam. $/$ pass. & $25.00(n=3)$ & $10,22(n-9)$ & $2.47(n-29)$ & $5,82(11-41)$ \\
\hline Querelleur & $25,00(n=3)$ & $13,57(n=7)$ & $2,43(n=29)$ & $6,16(n=39)$ \\
\hline \multirow[t]{2}{*}{ Ass. autre crime } & $25,00(n=4)$ & $12,76(n=25)$ & $3,89(n-10)$ & $11,74(n=39)^{* * *}$ \\
\hline & & & & $11,9 \%$ \\
\hline \multicolumn{5}{|l|}{ Mode de disposition*** } \\
\hline Pl. coup. acc.portée & - & $12,20(n=15)$ & $2,20(n=34)$ & $5,26(n=49)$ \\
\hline Pl. coup. acc. moindre & - & $13,07(n=14)$ & $3,51(n=30)$ & $6,55(n=44)$ \\
\hline \multirow[t]{2}{*}{ Procès } & $25,00(n=11)$ & $12.67(n=18)$ & $2,17(n=12)^{* *}$ & $12,91(n=41)^{* * *}$ \\
\hline & & & & $0,7 \%$ \\
\hline Total & $25,00(n=11)$ & $12,64(n=47)$ & $2,71(n=77)$ & $8,00(n=135)$ \\
\hline
\end{tabular}


Khi-carré, test-t, ou test de variance significatif à :

* $p=>0,01$ à 0,05 inclus.

$* * p=>0,00$ à 0,01 inclus.

$* * * p=0,00$

a : Pourcentage de valeurs manquantes.

b : Calcul du test-t impossible; tous les sujets ont le même délai.

c : Prudence face à la valeur de la tendance centrale et aux résultats d'analyses statistiques impliquant un petit nombre de sujets dans certaines cellules.

de tels antécédents. Néanmoins, nous savons que $62 \%$ des sujets condamnés qui en possèdent avaient commis des crimes de violence. Finalement, la loi considère qu'un homicide dans lequel l'auteur s'était muni d'une arme à feu, que la mort de la victime ait résulté ou non de l'emploi de cette arme, doit être considéré comme un meurtre (art. 230 C.c. ; Dubois, 1989). Considérant que plusieurs moyens ont pu être utilisés au cours d'un même événement en vue de mettre fin aux jours d'une personne, nous avons retenu celui qui s'avérait le plus fatal.

Parmi les caractéristiques du contrevenant et de la victime, dont la liste pourrait s'avérer longue, nous avons conservé le sexe de l'agresseur ainsi que le sexe de la victime. Également, la question des relations entre les protagonistes fut retenue. L'on distingue si l'agresseur connaissait la victime impliquée dans l'homicide, c'est-à-dire si les protagonistes entretenaient une relation quelconque, d'une quelconque intensité, depuis plus ou moins de 24 heures, ou si la victime était un pur étranger. Enfin, la variable du type d'homicide, qui est construite à partir du contexte relationnel entre les protagonistes au moment de l'homicide et des activités criminelles associées ou non à certains meurtres ${ }^{7}$, nous parut intéressante. Les trois types les plus fréquents sont les homicides familiaux ou passionnels (ce lien prime sur tout autre motif), les homicides commis au cours d'une querelle ou par vengeance personnelle, ainsi que les homicides commis durant la perpétration d'un autre crime par l'agresseur (par exemple un vol qualifié).

Puisque cette étude laisse une place importante à l'examen du cheminement des accusés à travers le processus judiciaire, nous avons cru bon, en dernier lieu, d'examiner l'influence que peut avoir le mode de disposition des causes au tribunal. Nous entendons par là la voie choisie au tribunal en vue de conclure les procédures, qu'il s'agisse d'un plaidoyer de culpabilité ou d'une reconnaissance de culpabilité suite à la tenue d'un procès.

Au tableau 2, les résultats permettent d'établir que sept facteurs ont une influence significative sur la détermination de l'accusation retenue à

7. Cette classification fut utilisée par Grenier (1993). 
l'issue du processus judiciaire. C'est ainsi (1) que le fait pour un accusé de subir un procès est un facteur fortement associé au verdict de meurtre (que ce soit au premier ou au second degré) plutôt que d'homicide involontaire coupable. Dans une moindre mesure, (2) le fait que l'homicide soit associé à la commission d'un autre crime par l'agresseur, (3) que ce dernier possède des antécédents judiciaires (4) et que des signes de préméditation soient présents entraînent aussi plus fréquemment une condamnation pour meurtre. Enfin, (5) le fait que l'agresseur ait tué une ou des personnes inconnues entraînent des probabilités légèrement supérieures qu'il soit condamné pour meurtre.

D'un autre côté, (1) le fait que l'accusé ait opté de plaider coupable, (2) le fait que ce dernier ait commis un homicide querelleur, familial ou passionnel, (3) ainsi que la présence d'événements déclencheurs peu avant l'homicide sont associés au choix de retenir envers le contrevenant une accusation d'homicide involontaire coupable en fin de procédures.

Le fait que l'auteur soit un homme semble affecter la sévérité de la peine dans son ensemble, puisqu'on note que ces auteurs sont environ deux fois plus punis comparativement aux accusées féminines. Mais il est à penser que les résultats auraient été plus significatifs s'il y avait eu davantage de sujets féminins.

Ces résultats tendent à corroborer ce que d'autres travaux de recherche ont établi. Cependant, les analyses bivariées ont aussi conduit à d'autres résultats plus inattendus. Ainsi, le genre de la victime n'a pas d'influence significative d'après les analyses bivariées. En outre, le fait que l'agresseur ait utilisé une arme à feu pour commettre l'homicide n'a pas suscité la réaction du tribunal à laquelle nous étions supposé nous attendre. En effet, bien qu'une proportion non négligeable d'accusés ayant eu recours à une arme à feu furent condamnés pour meurtre au premier degré, ce type d'arme mène avant tout à une accusation d'homicide involontaire coupable. La raison est que ce type d'arme a tendance à se rencontrer dans les homicides à caractère familial, conjugal ou passionnel, alors que de tels homicides conduisent le plus souvent à des accusations d'homicide involontaire coupable.

Pour la majorité des facteurs, l'jnfluence qu'ils exercent sur la sévérité globale des peines imposées se manifeste par l'entremise de leur impact sur le chef d'accusation décisif. Lorsque le chef d'accusation sur lequel le justiciable est condamné demeure, seul le mode de disposition des causes au tribunal se démarque avec des différences significatives de sévérité des peines sur le chef d'homicide involontaire coupable. C'est donc dire qu'un accusé de meurtre qui plaide coupable à une accusation réduite d'homicide involontaire coupable mérite une sentence généralement plus sévère que les contrevenants contre qui on a porté cette dernière accusation. 
Les analyses bivariées ont démontré l'existence de relations biunivoques une série de facteurs et les décisions judiciaires. Par l'entremise d'analyses multivariées, nous tenterons de faire ressortir quels facteurs permettent de prédire le chef d'accusation sur lequel porte le verdict de culpabilité, puis, en contrôlant par l'accusation décisive au moment du verdict, quels facteurs permettent de prédire la sévérité de la peine imposée. Ces analyses demeurent avant tout de nature exploratoire.

Nous avons choisi de tester un modèle d'analyse présentant l'ensemble des variables retenues, transformées en variables "fantômes", non sans avoir au préalable testé différents modèles dans le but d'étayer les résultats les plus probants. Les variables de l'âge de l'agresseur et de la victime furent ajoutées afin de vérifier leur influence, mais aussi pour stabiliser le modèle. La technique de la régression logistique, d'une part, et celle de la régression multiple, d'autre part, furent utilisées afin de répondre à nos questions. Compte tenu du nombre élevé de variables à insérer, la méthode d'entrée des variables stepwise fut privilégiée.

La technique de la régression logistique permet de prédire l'appartenance des sujets aux catégories de la variable dépendante à partir d'un ensemble de variables indépendantes. Elle a l'avantage de requérir des conditions d'utilisation moins contraignantes que l'analyse discriminante. En raison du peu de sujets condamnés pour meurtre au premier degré $(n=11)$, nous avons décidé de jumeler les catégories de meurtres. Les sujets seront donc distingués selon qu'ils sont accusés de meurtre ou d'homicide involontaire coupable.

Comme en témoigne le tableau 3 , il semble qu'il soit possible de prédire assez efficacement l'appartenance des sujets à l'accusation de meurtre par opposition à celle d'homicide involontaire coupable. Le modèle $d$ 'analyse, qui est statistiquement significatif $(p=0,02)$, rend compte d'un taux global de bonnes classifications de $76 \%$. Les sujets sont plus susceptibles d'être condamnés pour meurtre plutôt que pour homicide involontaire (1) s'ils ont tué durant la perpétration d'un autre crime ou s'ils ont commis un règlement de comptes; (2) s'ils ont subi un procès; (3) s'ils ont des antécédents judiciaires et, pour finir ; (4) si la victime est une femme.

Le premier résultat tend à confirmer qu'une dualité s'inscrit entre les homicides à caractère passionnel et ceux commis en particulier lors de la perpétration d'un autre crime par l'agresseur. Peu importent les facteurs contrôlés, le premier type d'homicides conduit systématiquement à des peines plus faibles que celles rendues pour le second. Le tribunal, tout comme la population, considère que ce dernier type d'homicide mérite une peine plus sévère, tout spécialement parce qu'il victimise un innocent ou un étranger (Daly et Wilson, 1988). Dans son étude sur l'homicide conjugal, Côté (1991, p. 36) a constaté que dans la majorité des cas, les conjoints 
meurtriers sont condamnés pour homicide involontaire coupable. Les plaidoyers de culpabilité sont nombreux et la défense de provocation est maintes fois invoquée dans ces affaires (voir aussi Cusson, 1987). Par conséquent, même en présence de signes de préméditation, un certain nombre de meurtres «passionnels» seront considérés en fin de procédures comme des homicides involontaires coupables. De ce fait, il se pourrait bien que la détermination de la peine ne soit pas strictement fonction du đegré de culpabilité et de gravité légales que reflète le crime.

Tableau 3

Les résuitats de la régression logistique : la variable dépendante est l'accusation sur laquelle porte le jugement

\begin{tabular}{|l|c|c|c|}
\hline Variable et codification & b & Signification & R \\
\hline 1. Homicide associé à un autre crime & 2,12 & 0,01 & 0,23 \\
2. Procès & 1,85 & 0,01 & 0,24 \\
3. Antécédents jud. (oui = 1) & 1,83 & 0,02 & 0,19 \\
4. Sexe de la vict. $(\mathrm{f}=1$ ) & 1,47 & 0,03 & 0,16 \\
(Constante) & $-3,44$ & 0,00 & \\
\hline Préméditation (oui = 1) & 0,11 & 0,07 & \\
Déclencheur imm. (oui = 1) & 0,54 & 0,00 & \\
Arme à feu (oui $=1$ ) & 0,33 & 0,00 & \\
Pl. coup. acc. moindre & 0,72 & 0,00 & \\
Âge de l'agresseur & 0,52 & 0,00 & \\
Âge de la victime & 0,72 & 0,00 & \\
Sexe de l'agress. $(\mathrm{f}=1$ ) & 0,74 & 0,00 & \\
Connaissance $(+24 \mathrm{~h}=1)$ & 0,30 & 0,00 & \\
Homicide familial & 0,51 & 0,00 & \\
\hline
\end{tabular}

Note :

Les catégories de la variable dépendante sont l'homicide involontaire coupable (0) et le meurtre (1).

$\mathrm{N}=\mathbf{7 4}$

Improvement 5,14 sig : 0,02

Pourcentage de bonnes classifications pour meurtre : $62 \%$

Pourcentage de bonnes classifications pour h.i.c. : $84 \%$

Pourcentage de bonnes classifications globales : $75,68 \%$

Amélioration sur le hasard : $52 \%$; sur le taux de base : $26 \%$

En suivant les accusés au cours du processus judiciaire, il est apparu, effectivement, que les sujets allant en procès étaient les plus lourdement taxés en ce qui concerne l'accusation retenue contre eux. Ainsi, les meurtriers condamnés à l'emprisonnement à perpétuité pour une période minimale de 25 ans ont en commun d'être tous passés par l'étape du procès criminel. En effet, il semblerait que la plupart des accusés allant en procès soient aux 
prises entre autres avec une accusation grave et un lourd dossier criminel, ce qui expliquerait que le mode de disposition soit en association avec la sévérité de la peine (Clarke et Kurtz, 1983). Cependant, les résultats des recherches sont controversés (voir entre autres Lafree, 1985).

Il n'est donc guère surprenant d'apprendre l'influence qu'ont les antécédents judiciaires sur la détermination du chef d'accusation à porter contre le prévenu. D'autres résultats de recherche abondent en ce sens : la présence d'antécédents judiciaires est de toute évidence un facteur aggravant (Vera Institute of Justice, 1977; Blumstein et coll., 1983; Clarke et Kurtz, 1983 ; Nakell et Hardy, 1987; Gottfredson et Gottfredson, 1988). Les contrevenants ayant un dossier criminel chargé ont de plus fortes probabilités d'être condamnés, et s'ils le sont, ils sont plus susceptibles de se voir imposer une peine sévère en comparaison de ceux n'en ayant pas ou dont le dossier est relativement peu important (Vera Institute of Justice, 1977).

Par ailleurs, un résultat quelque peu inattendu concerne l'influence qu'a le sexe de la victime sur l'accusation déterminante à l'issue des procédures. Ce facteur n'avait pas paru avoir d'impact significatif au cours des analyses bivariées. Deux études menées par Farrell et Swigert (1978, 1986) démontrent aussi que les auteurs d'homicide, peu importe leur genre, subissent un traitement pénal plus rigoureux lorsque la victime est une femme.

Pour terminer, nous aurions pu nous attendre à ce que des variables liées aux définitions légales des crimes qui permettent d'établir en partie le degré de gravité des actes, telles la présence de signes de préméditation, aient un rôle à jouer sur la détermination du chef d'accusation à conserver lors du verdict. Cependant, tel ne fut pas le cas.

Dans un deuxième temps, la technique de la régression multiple fut employée en vue d'identifier les facteurs ayant le plus d'impact sur la sévérité de la peine. Considérant les résultats précéđents, il a fallu procéder à des analyses qui contrôlent l'effet de l'accusation sur laquelle porte le jugement. La création d'une variable présentant un délai standardisé selon une mesure d'écart entre le délai individuel et le délai moyen pour chacun des trois groupes de délits d'homicide fut nécessaire. Cette variable se distribue normalement; la moyenne et la médiane équivalant à 1 (ce qui prévaut dans les cas dont le délai est de 25 ans pour meurtre au premier degré), la valeur minimale est de 0,004 et la maximale de 2,56.

Le tableau 4 permet de constater que les facteurs qui influencent la sévérité de la peine imposée sont peu nombreux et peu opérants quand la nature de l'accusation décisive lors du verdict demeure constante. Le modèle d'analyse, statistiquement significatif, permet d'expliquer une mince fraction de la variance $(12 \%)$ malgré le fait qu'une panoplie de facteurs soit considérée. D'ailleurs, la matrice d'intercorrélations ne présente guère de corrélations appréciables entre deux variables; la variable indépendante, 
notre principal intérêt, entretient de faibles relations linéaires avec les facteurs.

\section{Tableau 4}

Les résultats de la régression multiple : la variable dépendante est la sévérité de la peine prononcée (contrôle : l'accusation sur laquelle porte le verđict)

\begin{tabular}{|l|c|c|c|}
\hline Variable et codification & b & Beta & Signification \\
\hline 1. Pl. coup. acc. moindre & 0,33 & 0,28 & 0,02 \\
2. Sexe de l'agress. (f = 1) & $-0,56$ & $-0,26$ & 0,03 \\
(Constante) & 0,86 & & 0,00 \\
\hline Antécédents jud. (oui = 1) & & & 0,59 \\
Préméditation (oui = 1) & & & 0,25 \\
Déclencheur imm. (oui = 1) & & & 0,51 \\
Arme à feu (oui = 1) & & & 0,57 \\
Procès & & & 0,34 \\
Âge de l'agresseur & & & 0,10 \\
Âge de la victime & & & 0,40 \\
Sexe de la vict. (f $=1$ ) & & & 0,96 \\
Connaissance (+ 24 h= 1) & & & 0,83 \\
Homicide familial & & & 0,93 \\
Homicide associé à un autre crime & & & 0,25 \\
\hline
\end{tabular}

$\mathrm{N}=74$

$R^{2}=0,12 \quad F: 4,76 \quad$ sig. $F: 0,01$

Seuls deux facteurs ont apparemment un impact significatif sur l'ajustement à la hausse de la sévérité de la sentence : le fait de plaider coupable à une accusation moindre que celle initialement portée par la Couronne et le fait que l'agresseur soit un homme.

Lorsque l'ensemble des facteurs susceptibles de jouer sur la sévérité de la peine sont tenus constants, en l'occurrence les facteurs liês à la gravité de l'acte, une femme qui a commis un homicide est punie un peu moins sévèrement que ne le serait un homme. Farrell et Swigert (1986) ont étudié le traitement judiciaire de divers crimes dont le meurtre, et ils ont découvert que les meurtrières déclarées coupables recevaient un traitement sentenciel favorable puisqu'elles sont condamnées à purger des peines d'incarcération plus courtes que leurs semblables masculins. Ce constat est également rapporté par Zingraff et Thomson (1984) dans une recherche sur les délits d'homicide.

Toutes choses étant égales par ailleurs, le fait que le meurtrier soit un homme plutôt qu'une femme et le fait que la victime soit une femme plutôt qu'un homme conduit à un surcroît de sévérité. Il semble que les femmes jouissent d'un traitement légèrement favorable au tribunal : si elles sont 
meurtrières, elles sont traitées avec une relative clémence et si elles sont victimes, leur vie semble plus précieuse.

Un facteur qui se démarque à nouveau est le mode de disposition, en l'occurrence le plaidoyer de culpabilité à une accusation moindre que celle initialement portée. En contrôlant l'effet des autres variables, lorsque les sujets optent pour ce mode de disposition, la sentence est un peu plus sévère que celle qui est infligée aux accusés allant en procès ou à ceux qui plaident coupable à l'accusation portée par la Couronne. Comme nous l'avons constaté, cette situation se vérifiait particulièrement dans le cas des chefs d'homicides involontaires coupables. Les sujets ayant plaidé coupable à une accusation réduite étaient à l'origine accusés de meurtre. Leur cheminement a ceci de particulier que la conclusion des négociations favorables à une diminution du chef d'accusation a souvent lieu après que se soit terminée l'enquête préliminaire, peut-être devant la menace imminente du procès. Par conséquent, la sentence qu'ils ont reçue a tendance à être un peu plus élevée que celle qui fut imposée aux contrevenants n'ayant pas bénéficié d'une telle réduction de la gravité du chef d'accusation, afin de souligner la gravité factuelle associée à ces causes. Poirier, dans son étude sur la négociation des sentences (1987, p. 66), avait d'ailleurs fort bien expliqué ce phénomène.

\section{CONCLUSION}

Le but de cette étude était de mieux connaître l'intervention du tribunal de Montréal dans les causes d'homicide. Compte tenu des connaissances limitées sur le sujet, les deux objectifs spécifiques poursuivis ont été de cerner le processus d'attrition des inculpés au cours des procédures judiciaires suivies au tribunal et d'identifier les facteurs influençant les décisions pénales prises à leur endroit, notamment en ce qui concerne la détermination de la peine.

Les résultats montrent que le taux de condamnation pour les accusés traduits devant le tribunal pour homicide est élevé (80\%). Bien qu'à l'origine la plupart furent incriminés pour meurtre, le chef d'accusation qui domine à l'issue des procédures est l'homicide involontaire coupable $(55 \%)$. Cette situation est due en partie à la pratique de négociation entre avocats, qui a pour objet une diminution de la gravité qualitative de l'accusation par la Couronne en échange d'un plaidoyer de culpabilité par la défense. Enfin, une sentence carcérale fut infligée à presque tous les condamnés, dont la moitié ont quatre ans ou moins à purger avant d'être admissibles à une libération conditionnelle totale. La proportion de sujets condamnés qui se virent imposer l'emprisonnement à perpétuité pour un minimum de 25 ans avant d'être admissibles à une telle libération s'avère peu élevée, soit $8,5 \%$. 
Suite aux analyses explicatives, nous arrivons à la conclusion que les agents pénaux qui ont à intervenir dans des causes d'homicide à Montréal décident séquentiellement. Le processus débute par le choix que fait la Couronne de la première accusation à porter envers le contrevenant, ce qui aura des répercussions sur le mode de disposition de la cause adopté en cours de procédures (plaidoyer de culpabilité ou procès). L'évaluation des circonstances de l'acte et des caractéristiques personnelles et relationnelles des protagonistes a un impact sur la catégorie de délits d'homicide retenue à l'issue des procédures. Outre le fait qu'un homicide commis par un homme ou qu'un accusé plaidant coupable à une accusation réduite d'homicide involontaire coupable entraîne un surcroît de sévérité, la décision sentencielle qui est prise par la suite semble peu soupeser à nouveau les différents éléments propres à chaque cause d'homicide.

C'est đonc dire que dès le moment où est déterminé le chef d'accusation sur lequel portera le verdict, l'imposition d'une sentence tarifée sera la pratique courante, à quelques exceptions près. C'est dire qu'un quantum de peine sera déterminé à l'intérieur d'une fourchette étroite de peines (Cusson, 1987). S'il nous faut souligner l'influence qu'a le cheminement judiciaire suivi par les accusés sur les deux niveaux de décisions pénales étudiés, le fait cependant que les agents pénaux décident selon cette séquence nous porte à croire que s'il y a des négociations et des ententes conclues entre les parties dans les dossiers d'homicide, elles ne sont pas à proprement parler arbitraires. Quant à savoir si les peines imposées dans ces dossiers sont assez sévères, c'est une autre histoire. 


\section{BIBLIOGRAPHIE}

BLUMSTEIN, A., COHEN, J., MARTIN, S. E. et TONRY, M. H. (1983), Research on Sentencing : The Search for Reform, vol. I et vol. II, Washington D.C., National Academy Press.

BOYD, N. (1988), The Last Dance: Murder in Canada, Scarborough, Prentice-Hall Canada Inc.

CANADA (1990), Mise en liberté sous condition. Manuel à l'intention de la police, publié par la Commission nationale des libérations conditionnelles, Ottawa, Approvisionnements et Services Canada (catalogue JS92-34/1990F).

CLARKE, S. H. et KURTZ, S. T. (1983), « The Importance of Interim Decisions to Felony Trial Court Dispositions", The Journal of Criminal Law and Criminology, vol. 74, $n^{\circ} 2$, pp. 476-518.

COMMISSION DE RÉFORME DU DROIT DU CANADA (1984), Droit pénal : l'homicide, document de travail $n^{\circ} 3$, Ottawa, Approvisionnements et Services Canada.

CORDEAU, G. (1989), «Les homicides entre délinquants : une analyse des conflits qui provoquent les règlements de comptes ", Criminologie, vol. XXII, $n^{\circ} 2, \mathrm{pp} .13-34$.

COTÉ, A. (1991), La Rage au caur, rapport de recherche sur le traitement judiciaire de I'homicide conjugal au Québec, Baie-Comeau, Regroupement des femmes de la Côte-Nord.

COUSINEAU, M.-M. (1992), Processus décisionnel et détermination des trajectoires judiciaires : analyse du cheminement d'une cohorte de justiciables, thèse de doctorat (Sociologie), Université du Québec à Montréal, Département de sociologie.

CUSSON, M. (1987), Pourquoi punir?, Paris, Dalloz.

DALY, M., et WILSON, M. (1988), Homicide, New York, Aidine de Gruyter.

DUBOIS, A. (1989), Code criminel annoté et lois connexes, Cowansville, les Éditions Yvon Blais inc.

FARREL.L, R. A., et SWIGERT, V. L. (1978), «Prior Offense Record as a Self-Fulfilling Prophecy ", Law and Society Review, vol. 12, $\mathrm{n}^{\circ} 3$, pp. 437-454.

FARRELL, R. A., et SWIGERT, V. L. (1986), «Adjudication in Homicide : An Interpretive Analysis of the Effects of Defendant and Victim Characteristics ", Journal of Research in Crime and Delinquency, vol. 23, $\mathrm{n}^{\circ} 4$, pp. 349-369.

FIGUEIRA-McDONOUGH, J. (1985), "Gender Differences in Informal Processing ", Journal of Research in Crime and Delinquency, vol. 22, $\mathrm{n}^{\circ} 2$, pp. 101-133.

GOTTFREDSON, M. R., et GOTTFREDSON, D. M. (1988), Decision Making in Criminal Justice. Toward the Rational Exercise of Discretion, New York, Plenum Press.

GRAVEL, S. et BARIL, M. (1990), La pratique de la négociation de plaidoyer au Palais de justice de Montréal, Université de Montréal, Centre international de criminologie comparée.

GRENIER, S. (1993), L'Évolution des catégories d'homicides au Québec de 1954 à 1989, mémoire de man̂trise ès Sciences (Criminologie), Université de Montréal, École de criminologie.

KERR, N. L. (1978), "Severity of Prescribed Penalty and Mock Jurors' Verdicts", Journal of Personality and Social Psychology, vol. $36, n^{\circ} 12$, pp. 1431-1442. 
LAFREE, G. (1985), "Adversarial and Non-adversarial Justice ", Criminology, vol. 23, $n^{\circ} 2$, pp. 289-312.

McCARTHY, B. R. et LINDQUIST, C. A. (1984), « Factors Influencing Sentences for Violent Offenses: An Exploratory Comparison of Crime-specific and Undifferentiated Research Strategies », Criminal Justice Review, vol. 9, $\mathrm{n}^{\circ}$ 2, pp. 37-46.

NADIN-DAVIS, R. P. (1982), Sentencing in Canada, Ottawa, The Carswell Company Limited.

NAKELL, B. et HARDY, K. A. (1987), The Arbitrariness of the Death Penalty, Philadelphie, Temple University Press.

POIRIER, R. (1987), «La négociation des sentences du point de vue des avocats de la défense ", Criminologie, vol. XX, $\mathrm{n}^{\circ} 2$, pp. 57-68.

QUÉBEC (1989), Lois et règlements concernant les personnes incarcérées, Québec, Les Publications du Québec.

RUBY, C. C. (1987), Sentencing, Toronto et Vancouver, Butterworths.

SILVERMAN, R. A. et KENNEDY, L. (1993), Deadly Deeds : Murder in Canada, Ontario, Nelson Canada.

STATISTIQUE CANADA (1990), L'Homicide au Canada (1989), Juristat, Bulletin de service, Statistique Canada, Centre canadien de la statistique juridique, vol. 10, $\mathrm{n}^{\circ}$ 14, Ottawa, Approvisionnements et Services Canada (catalogue 85-002).

STIPAK, B. et McDAVID, J. C. (1983), « Statistical Procedures for Analyzing Factors Affecting Judicial Sentences Using Simultaneously Derived Crime Seriousness and Sentence Severity Scales", International Journal of Comparative and Applied Criminal Justice, vol. 7, n 1, pp. 21-34.

TREMBLAY, P., GRAVEL, S. et CUSSON M. (1987), «Équivalences pénales et solutions de rechange à l'emprisonnement : la métrique pénale implicite des tribunaux criminels ", Criminologie, vol. XX, $\mathrm{n}^{\circ} 2, \mathrm{pp} .69-88$.

VERA INSTITUTE OF JUSTICE (1977), Felony Arrests: Their Prosecution and Disposition in New York City's Courts, New York, Vera Institute of Justice.

ZINGRAFF, M. et THOMSON, R. (1984), «Differential Sentencing of Women and Men in the U.S.A.", International Journal of the Sociology of Law, vol. 12, pp. 401-413. 